\title{
TIME-HOPPING MULTICARRIER CODE-DIVISION MULTIPLE-ACCESS: SYSTEM OUTLINE AND PERFORMANCE
}

\author{
Lie-Liang Yang \\ School of ECS, University of Southampton, SO17 1BJ, UK
}

\begin{abstract}
A time-hopping multicarrier code-division multiple-access (TH/MC-CDMA) scheme is proposed and investigated. In the proposed TH/MC-CDMA each information symbol is transmitted by a number of time-domain pulses with each timedomain pulse modulating a subcarrier. The transmitted information at the receiver is extracted from one of the, say $M$, possible time-slot positions, i.e., assuming that $M$-ary pulse position modulation is employed. Specifically, in this contribution we concentrate on the scenarios such as system design, power spectral density (PSD) and signal detection. The error performance of the TH/MC-CDMA system is evaluated, when the channel that each subcarrier signal experiences is modeled as flat Nakagami- $m$ fading in addition to the additive white Gaussian noise (AWGN). According to our analysis and results, it can be shown that the TH/MC-CDMA signal is capable of providing a near ideal PSD, which is flat over the system bandwidth available, while decreases rapidly beyond that bandwidth. Explicitly, signals having this type of PSD is beneficial to both broadband and ultra-wide bandwidth (UWB) communications. Furthermore, our results show that, when optimum user address codes are employed, the single-user detector considered is near-far resistant, provided that the number of users supported by the system is lower than the number of subcarriers used for conveying a information symbol.
\end{abstract}

\section{INTRODUCTION}

In time-hoping (TH) UWB communications data is transmitted using extremely short time-domain pulses with duration less than 1 ns. Multiple users in UWB systems are usually supported with the aid of the TH techniques. Since UWB systems usually have a bandwidth on the order of gigahertz, a typical characteristic of UWB channels is frequency-selective fading, which may result in a huge number of resolvable multipath components at the receiver [2]. Consequently, the receiver's complexity of the TH UWB systems might be extremely high, when using RAKE-like receivers. This is because the RAKE receiver requires a very high number of fingers and each finger requires corresponding channel estimation, in order to achieve a high multipath combining efficiency.

In this contribution, a TH multicarrier CDMA (TH/MC CDMA) scheme is proposed, where data is transmitted using relatively high-duration time-domain pulses with the aid of $M$-ary pulse position modulation (MPPM), where information is extracted from one of the $M$ possible time-slot positions. In TH/MC-CDMA each symbol is transmitted using a number of time-domain pulses with each time-domain pulse modulating a subcarrier. Since each subcarrier only occupies a fraction of the system bandwidth, the time-domain pulses hence have a relatively high duration. Consequently, even the total system bandwidth is extremely high, the subcarrier signals may be designed so that they are not too dispersive and only a low number of independent multipath components appear at the receiver. In TH/MC-CDMA system diversity gain may be achieved by combining the independently faded multipath components or/and by transmitting the same information symbol using several subcarriers, which are combined at the receiver. In TH/MC-CDMA the transmitter does not conflict the problem of nonlinear fluctuation resulted from the high peak-to-average factor, since in the TH/MC-CDMA transmitter only one of the subcarrier signals is activated at any timeinstant. However, fast-Fourier transform (FFT) assisted multicarrier demodulation technique can still be invoked for demodulating multiuser TH/MC-CDMA signals. In fact, in TH/MCCDMA single-user or multiuser detection can be carried out after a single FFT-assisted multicarrier demodulation device.

In this contribution we consider the design and performance issues of the TH/MC-CDMA. Specifically, a synchronous TH/MC-CDMA communication scheme is considered and the parameter design in the TH/MC-CDMA system is investigated. It can be shown that, by appropriately selecting the system parameters, the TH/MC-CDMA signal can appear a Gaussian noise-like flat PSD over the system bandwidth available. Furthermore, the PSD's outband sidelobes of TH/MC-CDMA signals decrease much more rapidly, in comparison to a corresponding single-carrier TH-CDMA signals, which has the same system bandwidth as the TH/MC-CDMA signals. In this contribution the error performance of a single-user detector is investigated, when the subcarrier channels are modeled as flat Nakagami- $m$ fading in the presence of AWGN. The single-user detector is designed based on the frequency-time matrix principles, which forms the extension of the time-frequency matrixassisted detection in fast frequency-hopping (FFH) systems [6].

\section{TIME-HOPPING MULTICARRIER CDMA SCHEME}

\section{A. Time-Hopping Multicarrier CDMA Signal}

The transmitter schematic of the $k$ th user is shown in Fig. 1 for the TH/MC-CDMA system. At the transmitter side, the input binary data having a period $T_{b}$ and a rate $R_{b}$ is serial-to-parallel (S/P) converted to $U$ parallel streams. During a frame interval of $T_{f}$ seconds, each stream transmits $b=\log _{2} M$ number of bits referred to as a symbol. Hence, each frame transmits $U$ symbols in parallel and both the symbol duration and the frame duration are $T_{s}=T_{f}=U b T_{b}$ seconds. Let the total number of subcarriers invoked in the TH/MC-CDMA system is $U L$ and, hence, each stream is transmitted on $L$ number of subcarriers. Let the TH address code of user $k, 1 \leq k \leq K$, be expressed as $\mathbf{a}_{k}=\left[a_{0}^{(k)}, a_{1}^{(k)}, \ldots, a_{L-1}^{(k)}\right]$, where $a_{l}^{(k)} \in G F(M), l=$ $0,1, \ldots, L-1$, and $G F(M)$ denotes a Galois field obey $M=$ $2^{b}$. Furthermore, let $x_{k} \in G F(M)$ be a $b$-bit symbol of one of the $U$ streams, say $u$, which will be transmitted by the $L$ number of subcarriers corresponding to the $u$ th stream. Then, $x_{k}$ is signatured by the $k$ th user's TH address code, which can be expressed as

$$
\mathbf{Y}_{k}=\left[y_{k}(0), y_{k}(1), \ldots, y_{k}(L-1)\right]=x_{k} \cdot \mathbf{1} \oplus \mathbf{a}_{k}
$$

where 1 represents a unit vector of length $L, y_{k}(l), 0 \leq l \leq$ $L-1$ are elements of $G F(M)$, and $\oplus$ denotes the addition 


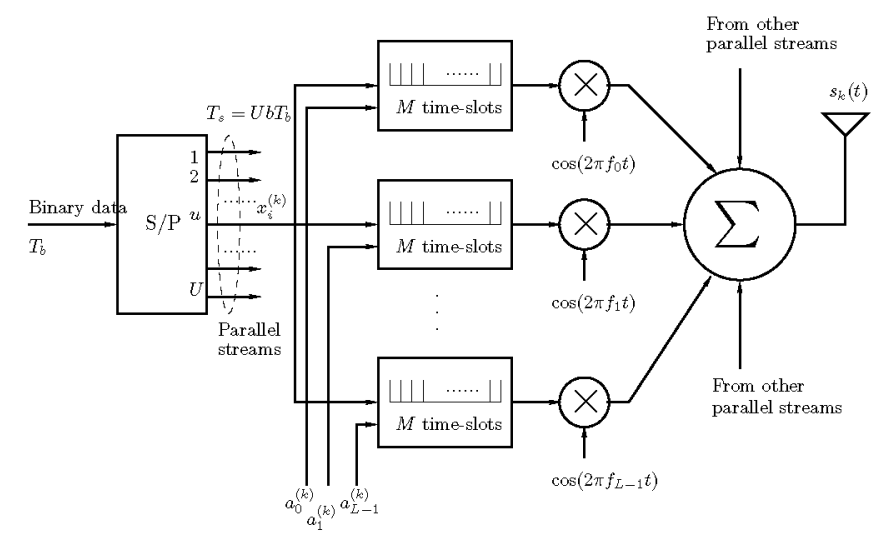

Figure 1: The $k$ th user's transmitter schematic for the time-hopping multicarrier CDMA.

operation in $G F(M)$. As shown in Fig.1, for each subcarrier branch, there are $M=2^{b}$ time-slots within a frame duration of $T_{f}$ seconds. After the signature operation of (1), one of the $M$ time-slots corresponding to each subcarrier branch is then activated by one of the elements of $\mathbf{Y}_{k}$. Specifically, for the $u$ th stream transmitted by $L$ number of subcarriers, the $y_{k}(l)$ th time-slot of the $l$ th subcarrier will be activated for transmission, while the other $(L-1)$ time-slots of the $l$ th subcarrier cease transmission. After the above described TH operation, the $l$ th subcarrier branch modulates a subcarrier frequency $f_{l}$ for $l=0,1, \ldots, L-1$ within the active time-slot. Finally, as shown in Fig. 1, the $U L$ subcarrier-modulated signals are added in order to form the transmitted signal. For the $u$ th stream, the transmitted signal can be expressed as

$$
\begin{array}{r}
s_{k}(t)=\sqrt{\frac{2 P M}{L}} \sum_{l=0}^{L-1} \psi_{T_{h}}\left(t-y_{k}(l) T_{h}\right) \\
\times \cos \left(2 \pi\left(f_{c}+f_{l}\right) t+\varphi_{l}^{(k)}\right)
\end{array}
$$

where $P$ represents the transmitted power of the $b$-bit symbol $x_{k}, f_{c}$ represents the carrier frequency, $\psi_{T_{h}}(t)$ represents a pulse-waveform defined within $\left[0, T_{h}\right)$ and it is normalized so that $\int_{0}^{T_{h}} \psi_{T_{h}}^{2}(t) d t=T_{h}$. Note that, equation (2) considers only one of the $U$ parallel streams. The other parallel streams can be considered in a similar way and have the same transmitted signal form as (2), except that the transmitted $b$-bit symbols and the subcarrier frequencies need to be changed correspondingly. However, for synchronous TH/MC-CDMA systems considered in this contribution, the performance evaluation can be carried out when considering only one of the parallel streams. Hence, unless otherwise mentioned, only one of the parallel streams is considered for the sake of simplicity in our forthcoming discourse.

\section{B. Modulation Parameters}

Let us assume a total $W_{s} \mathrm{~Hz}$ of bandwidth that the TH/MCCDMA system can exploit. Let us also assume that the input binary data rate be $R_{b}=1 / T_{b}$. Below we investigate the constraints associated with the parameter $M$ of the number of time-slots per TH/MC-CDMA frame, the parameter $L$ of the number of subcarriers per parallel stream used for transmission

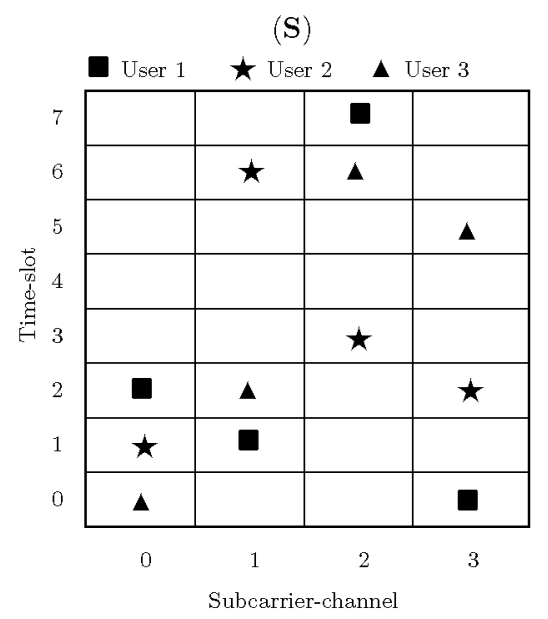

Figure 2: Pictorial representation of transmitted signals for a threeuser TH/MC-CDMA system using $M=8$ time-slots per symbol and $L=4$ subcarriers.

and the parameter $W_{t h}$ of the TH bandwidth, which is also the null-to-null bandwidth per subcarrier signal. The pictorial representation of the transmitted signal in a three-user TH/MCCDMA system using $M=8$ time-slots per frame and $L=4$ subcarriers is shown in Fig. 2, where we assumed that $x_{k}$ in (1) are $x_{1}=3, x_{2}=5$ and $x_{3}=7$ and the TH address codes are $[1,2,4,3]$ for user $1,[4,3,6,7]$ for user 2 and $[7,5,1,2]$ for user 3 .

Firstly, when the TH duration is assumed to be $T_{h}$ seconds, then the frequency spacing between two adjacent TH tones should not be less than $R_{h}=1 / T_{h} \mathrm{~Hz}$, which is the constraint assumed in this contribution. Consequently, for a TH/MCCDMA system using $U L$ number of subcarriers, we have the relationship among the parameters $W_{s}, U$ and $L$ expressed as $W_{s} \approx U L R_{h}=U L / T_{h}$. Secondly, we have known that the frame duration is $T_{f}=U b T_{b}$. Hence, the number of TH slots $M$ obeys $M=2^{b}=T_{f}=U b T_{b} / T_{h}$. Consequently, it can be shown that we have $M=2^{b} / b=T_{b} W_{s} / L=W_{s} / R_{b} L$.

\section{Power Spectral Density}

The PSD of the TH/MC-CDMA signals can be derived using the similar approaches in [8]. Specifically, for real pulse shape waveform of $\psi_{T_{h}}(t)$, the PSD can be expressed as

$$
\begin{array}{r}
S_{T}(f)=\frac{P M}{2 L T_{f}}\left\{\sum_{u=1}^{U} \sum_{m=0}^{L-1} \Psi^{2}\left(f-f_{u m}\right)\right. \\
\left.+\sum_{u=1}^{U} \sum_{m=0}^{L-1} \Psi^{2}\left(f+f_{u m}\right)\right\}
\end{array}
$$

where $\Psi(f)$ represents the Fourier transform of the timedomain pulse $\psi_{T_{h}}(t)$, i.e., we have $\Psi(f)=\mathcal{F}\left\{\psi_{T_{h}}(t)\right\}$. Explicitly, Equation (3) shows that the PSD of the TH/MCCDMA signal is simply the sum of the basic pulse's PSD, $\Psi^{2}(f)$, translated to all subcarrier frequencies and weighted by a constant of $\frac{P M}{2 L T_{f}}$.

As an example, let us consider the $T_{h}$-width pulse $\psi_{T_{h}}(t)=$ $\sqrt{2} \cos \left(\frac{\pi t}{T_{h}}\right) \prod\left(\frac{t}{T_{h}}\right)$ [3], where $\prod\left(\frac{t}{T_{h}}\right)$ represents a rectan- 


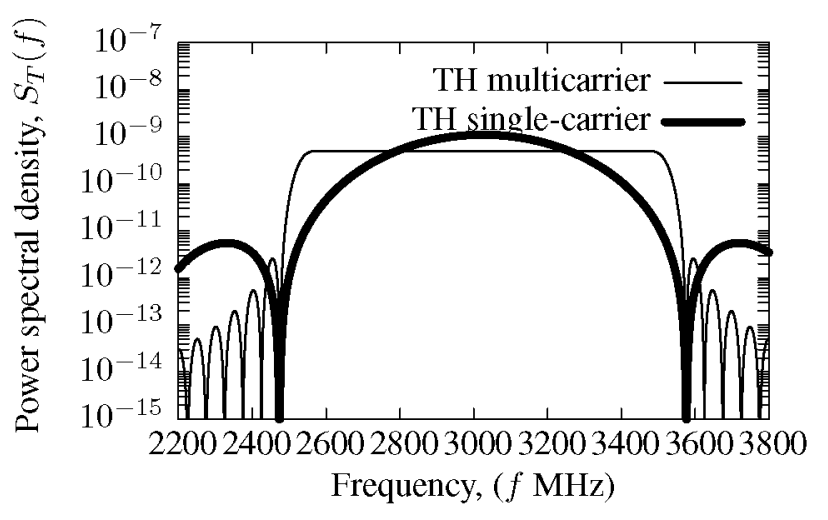

Figure 3: Power spectral density (PSD) centered at $3.025 \mathrm{GHz}$ for both multicarrier and single-carrier based TH signals occupying a bandwidth of about $1.1 \mathrm{GHz}$.

gular waveform defined in $\left[0, T_{h}\right)$. The Fourier transform of $\psi_{T_{h}}(t)$ can be expressed as [3]

$$
\Psi(f)=\frac{2 \sqrt{2} T_{h}}{\pi} \times \frac{\cos \left(\pi T_{h} f\right)}{1-4 T_{h}^{2} f^{2}}
$$

Upon substituting (4) into (3) and considering $T_{f}=M T_{h}$, the corresponding PSD can be expressed as

$$
\begin{array}{r}
S_{T}(f)=\frac{4 P T_{h}}{L \pi^{2}}\left\{\sum_{u=1}^{U} \sum_{m=0}^{L-1} \frac{\cos ^{2}\left[\pi T_{h}\left(f-f_{u m}\right)\right]}{\left[1-4 T_{h}^{2}\left(f-f_{u m}\right)^{2}\right]^{2}}\right. \\
\left.+\sum_{u=1}^{U} \sum_{m=0}^{L-1} \frac{\cos ^{2}\left[\pi T_{h}\left(f+f_{u m}\right)\right]}{\left[1-4 T_{h}^{2}\left(f+f_{u m}\right)^{2}\right]^{2}}\right\}
\end{array}
$$

The PSD of (5) for TH/MC-CDMA signals is shown in Fig.3, when $U L=20$ number of subcarriers are assumed. As shown in Fig.3, the total system null-to-null bandwidth is about $W_{s}=1.1 \mathrm{GHz}$ and the centered frequency is $f_{c}=3.025 \mathrm{GHz}$. Note that, Fig.3 only shows the PSD corresponding to the positive frequency band, which were computed from the first summation of (5). According to (5) it is easy to know that the PSD corresponding to the negative frequency band has the same shape as that of the positive frequency band, but is centered at the frequency $f_{c}=-3.025 \mathrm{GHz}$. In Fig.3 we also show the PSD of the single-carrier-based TH-CDMA signals, which occupies an equivalent bandwidth of $1.1 \mathrm{GHz}$. As shown in Fig. 3 TH/MC-CDMA signals have a flat, Gaussian noise-like PSD over the available frequency band and the transmitted power is near-ideally distributed over the whole system bandwidth. This PSD characteristic is very important to broadband or UWB [1] communications, since with the Gaussian noise-like PSD the resource of bandwidth can be exploited with the highest efficiency. Furthermore, as shown in Fig.3, the PSD's sidelobes of the TH/MC-CDMA signals decrease much more rapidly, than that of the corresponding single-carrier TH-CDMA signals.

\section{Receiver Model}

We assume a free-space propagation channel with Nakagami$m$ fading and AWGN. Consequently, when $K$ TH/MC-CDMA signals obeying the form of (2) are transmitted synchronously over this type of channels, the received complex low-pass

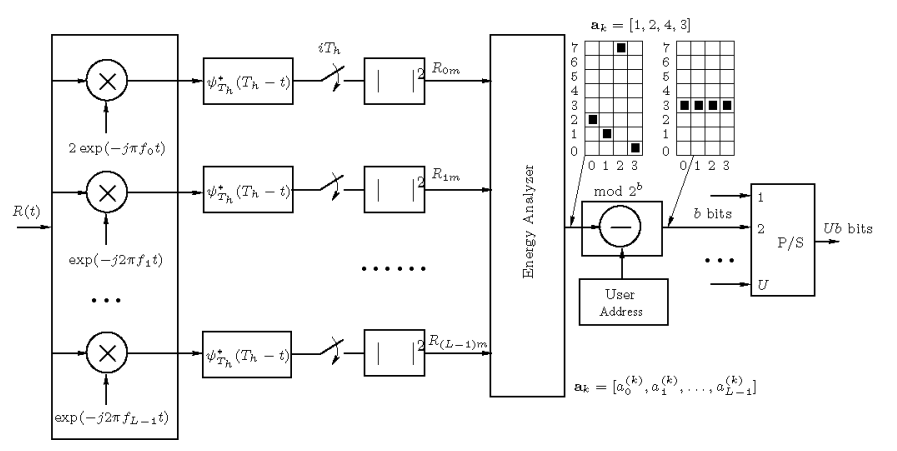

Figure 4: Receiver block diagram of the TH/MC-CDMA scheme.

equivalent signal can be expressed as

$$
\begin{array}{r}
R(t)=\sum_{k=1}^{K} \sqrt{\frac{2 P M}{L} \sum_{l=0}^{L-1} \alpha_{l}^{(k)} \psi_{T_{h}}\left(t-y_{k}(l) T_{h}\right)} \\
\quad \times \exp \left(j\left[2 \pi f_{l} t+\varphi_{l}^{(k)}\right]\right)+N(t)
\end{array}
$$

where $N(t)$ represents the complex low-pass equivalent AWGN with zero mean and single-sided power spectral density of $2 N_{0}$, while $\alpha_{l}^{(k)}$ represents an independent identically distributed (i.i.d) Nakagami- $m$ random variable with a probability density function (PDF) given by[4]

$$
p_{\alpha_{l}^{(k)}}(R)=\frac{2 m^{m} R^{2 m-1}}{\Gamma(m) \Omega^{m}} e^{(-m / \Omega) R^{2}}
$$

where $\Gamma(\cdot)$ is the gamma function, $m$ is the Nakagami- $m$ fading parameter and $\Omega=E\left[\left(\alpha_{l}^{(k)}\right)^{2}\right]$.

Due to the time-hopping characteristic, we assume that the receiver is unable to estimate the fading amplitudes $\left\{\alpha_{l}^{(k)}\right\}$ and the phases $\left\{\varphi_{l}^{(k)}\right\}$, except the synchronization with the received signal. The receiver schematic of the TH/MC-CDMA system considered is shown in Fig.4, which essentially represents an energy assisted detector. The receiver constitutes a symbol-by-symbol or frame-by-frame detector. Specifically, at the receiver of Fig.4, the received signal is first multicarrier demodulated with respect to each of the $L$ subcarriers used for the transmission of the $M=2^{b}$-ary symbol $x_{k}$. Then, for each subcarrier branch, the output signal after subcarrier demodulation is input to a matched-filter, which matches to the time-domain pulse $\psi_{T_{h}}(t)$ transmitted within a time-slot. As shown in Fig.4, the matched-filter's output is sampled and then squared associated with each of the $M$ number of time-slots in a frame, in order to generate a decision variable for detecting the corresponding energy level. Since there are $L$ subcarriers and each subcarrier conveys $M$ number of time-slots, we are provided with a total of $M L$ number of outputs during a frame duration of $T_{f}=U b T_{b}$ seconds. As shown in Fig.4, the timeslots of $L$ subcarriers in the context of each frame are analyzed in order to determine, which time-slots and, hence, which $b$-bit symbol $x_{k}$ was transmitted by invoking the detection scheme described below.

In TH/MC-CDMA systems, as described previously in the context of the transmitter of Fig.1, a user address is employed 


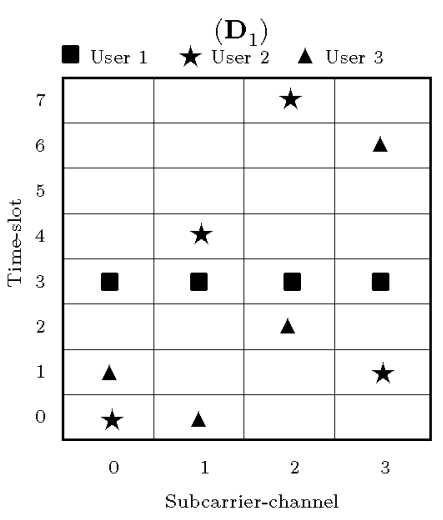

Figure 5: Frequency-time matrix description for the detection of the 8 -ary symbol $x_{1}=3$ transmitted by user 1 using the transmitted frequency-time matrix of Fig. 2 .

as a unique signature sequence, in order to signal the time-slots conveying a $b$-bit symbol of the user considered, as shown in (1). Following energy detection in terms of each time-slot of the $L$ subcarriers, the sequence $\mathbf{Y}_{k}$ of (1) can be recovered by the receiver, provided that the channel is sufficiently good. By performing the subtraction operation of the unique user address $\mathbf{a}_{k}$ of the $k$ th user from $\mathbf{Y}_{k}$ on a subcarrier-by-subcarrier basis, we arrive at

$$
x_{k} \cdot \mathbf{1}=\mathbf{Y}_{k} \ominus \mathbf{a}_{k}
$$

which allows us to recover the transmitted symbol $x_{k}$ of user $k$. In (8) $\ominus$ represents the subtraction operation in $G F(M)$ [5].

The detection operation of the TH/MC-CDMA system can be understood by means of frequency-time matrices ${ }^{1}$ having $M$ rows and $L$ columns, where the $M$ rows correspond to the $M$ distinct time-slots of a frame, while the $L$ columns represent the $L$ number of subcarriers. We refer to the elements of the frequency-time matrices as frequency-time elements. The frequency-time matrix has the same feature as those shown in Fig. 2, where we assumed that a range of the frequency-time elements were activated by the transmitters of user 1,2 and 3 . In order to make use of the frequency-time matrices for facilitating the detection at the receiver, the first step is to form a received frequency-time matrix $\mathbf{R}$ based on the decision variables. Specifically, let us assume that $h$ is a decision threshold. Then, whenever the energy-related decision variable, say $R_{l m}$ in Fig.4, exceeds the threshold $h$, the corresponding frequencytime element at row $m$ column $l$ is flagged by a logical 1 (such as the markers in Fig. 2), otherwise, by a logical 0 (null).

Let the transmitted frequency-time matrix associated with three users be represented by Fig.2. Provided that no channel impairments are imposed, all elements of the received frequency-time matrix, say $\mathbf{R}$, are received correctly, i.e., the received frequency-time matrix $\mathbf{R}$ is the same as the transmitted frequency-time matrix $\mathbf{S}$ of Fig.2. Since the unique TH address assigned to user $\mathbf{1}$ is $\mathbf{a}_{1}=[1,2,4,3]$, for the matrix $\mathbf{R}$ of Fig.2, the resultant frequency-time matrix $\mathbf{D}_{1}$ obtained by subtraction of the unique user code address $\mathbf{a}_{1}$ from $\mathbf{R}$ on a column-by-column basis according to (8) is shown in Fig.5.

${ }^{1}$ The frequency-time matrix described here is inspired by the concept of time-frequency matrix [6], which has been applied for describing the detection of fast frequency-hopping (FFH) signals.
Let us employ a simple Majority Logic Decision (MLD) based decoder, which opts for deciding the particular 8-ary symbol corresponding to the specific row having the highest number of non-zero entries, for the sake of providing an estimate to the transmitted symbol $x_{1}$ of user 1 . Hence, based on the frequency-time matrix $\mathbf{D}_{1}$ of Fig.5, we infer that the transmitted symbol $x_{k}=3$ has been correctly decoded with the aid of unambiguous MLD. Furthermore, it can be shown that the transmitted symbols $x_{2}=5$ by user 2 and $x_{3}=7$ by user 3 can also be detected correctly by using the same approach described above.

\section{EXAMPLES OF PERFORMANCE RESULTS}

In this Section the performance of the TH/MC-CDMA systems using optimum user address codes [5] was evaluated and compared for a range of parameters.

Fig. 6 shows the BER performance of the TH/MC-CDMA system versus the SNR per bit, $E_{b} / N_{0}$ and versus the threshold, $h$, when communicating over the Nakagami- $m$ fading channels having the fading parameter of $m=1.2$. It is clear from Fig. 6 that an inappropriate choice of the detection threshold $h$ may lead to significant increase of the BER. For any given threshold $h$, the BER decreases, as the SNR per bit increases. By contrast, for any given SNR per bit value there exists an optimal choice of the threshold $h$, which minimizes the BER. Furthermore, from Fig. 6 we observe that the optimum threshold value slightly increases, as increasing the SNR per bit value. This property may be used for predicting the optimum thresholds at different SNR values. Hence, in our further investigations we assumed that the optimum threshold was employed, whenever the detection threshold $h$ was invoked.

Fig. 7 shows the bit error probability of the TH/MC-CDMA systems with respect to supporting various number of users, when communicating over the Nakagami- $m$ fading channels associated with the fading parameter $m=1.5$. From the results Fig. 7, we observe that, for a given SNR per bit value, the BER increases, when the TH/MC-CDMA system supports more users. We can also observe that, if $K \leq L=4$ number of users is supported, the BER decreases without error floor, as the SNR per bit value increases. By contrast, when the number of users supported is higher than the value of $L=4$, we can clearly observe the error floors in these figures. Hence, for synchronous TH/MC-CDMA systems using optimum address codes, the considered single-user based detector is near-far resistance, provided that the number of users supported by the system does not exceed the number of subcarriers conveying a $M$-ary symbol.

In Fig. 8 we show the influence of the fading parameter $m$ and the number of subcarriers $L$, which are invoked for transmitting the same 5-bit ( $M=32$ ) symbol, on the bit error probability. Note again that the case of $m=1$ corresponds to the Rayleigh fading channel, while that the cases of $m=1.5,3$ represent the channels, such as Rician fading channels, that are less faded than the Rayleigh fading channels. From the results, we observe that, for the TH/MC-CDMA scheme assuming a given SNR per bit value, the BER decreases when $m$ or $L$ increases. Furthermore, as shown in Fig. 8, the SNR gain corresponding to a low $m$ value, such as $m=1$, is more significant than that corresponding to a high $m$ value, such as $m=3$, when increasing the number of subcarriers $L$. Hence, in TH/MC-CDMA systems, when the wireless channels experience severe fading, a high number of subcarriers may be em- 
ployed for conveying the same information symbol, in order to achieve a high diversity gain and, ultimately, to achieve a low bit error probability.

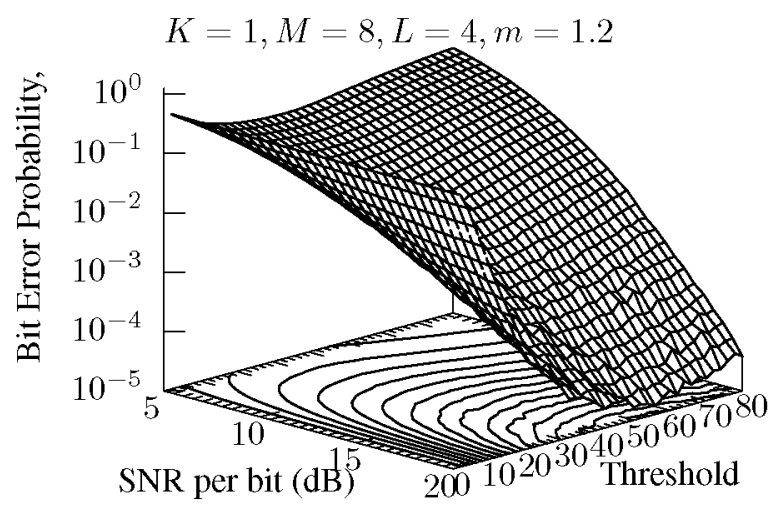

Figure 6: BER versus the SNR per bit, $E_{b} / N_{0}$, and versus the threshold, $h$, performance for the TH/MC-CDMA systems supporting $K=1$ user, when communicating over Nakagami- $m$ fading channels having the fading parameter $m=1.2$.

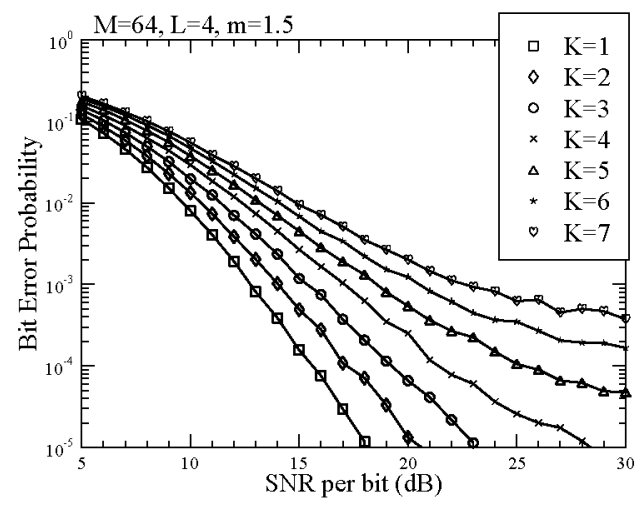

Figure 7: BER versus SNR per bit performance for the TH/MCCDMA system using parameters of $M=64, L=4$, when communicating over Nakagami- $m$ fading channels having the fading parameter $m=1.5$.

In conclusions, in this contribution we have proposed and investigated a novel multiple-access scheme, namely the TH/MC-CDMA scheme, which is based on the time-hopping, multicarrier modulation and CDMA techniques. From our analysis and results we find that TH/MC-CDMA is capable of providing a range of advantages. Specifically, the TH/MCCDMA signals have a flat, Gaussian noise-like PSD over, probably, a very wide bandwidth. The PSD outband sidelobes decrease much more rapidly, than that of a corresponding singlecarrier TH-CDMA signal. In TH/MC-CDMA, due to the timehopping characteristic, each subcarrier is only activated for a fraction of the frame time-duration. Hence, the nonlinear fluc-

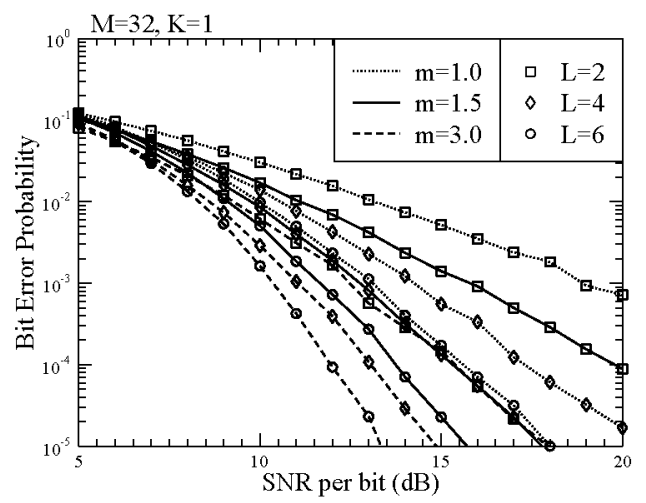

Figure 8: BER versus SNR per bit performance for the TH/MCCDMA system, when communicating over Nakagami- $m$ fading channels with various fading parameters.

tuation due to the high peak-to-average factor in conventional multicarrier systems can be significantly mitigated. Furthermore, in TH/MC-CDMA receiver the processing rate is on the order of the time-hopping rate $R_{h}$, which is significantly less demanding than the demodulation during each chip interval, which would be necessitated in direct-sequence CDMA (DSCDMA). Based on the above-mentioned arguments, it can be shown that TH/MC-CDMA constitutes a promising multipleaccess scheme, which is capable of supporting broadband or UWB communications.

\section{ACKNOWLEDGEMENT}

The author would like to acknowledge with thanks the financial assistance from EPSRC of UK.

\section{REFERENCES}

[1] M. Z. Win and R. A. Scholtz, "Ultra-wide bandwidth time-hopping spread-spectrum impulse radio for wireless multiple-access communications," IEEE Trans. on Commun., vol. 48, pp. 679-691, Apr. 2000.

[2] M. Z. Win and R. A. Scholtz, "On the robustness of ultra-wide bandwidth signals in dense multipath environments," IEEE Commun. Letters, vol. 2, pp. 51-53, Feb. 1998.

[3] R. E. Ziemer and R. L. Peterson, Digital Communications and Spread Spectrum Systems. New York: Macmillan Publishing Company, 1985.

[4] L.-L. Yang and L. Hanzo, "Performance of generalized multicarrier DSCDMA over Nakagami- $m$ fading channels," IEEE Trans. on Commun., vol. 50 , pp. $956-966$, June 2002.

[5] G. Einarsson, "Address assignment for a time-frequency-coded, spreadspectrum system," Bell System Technical Journal, vol. 59, pp. 12411255, September 1980 .

[6] D. J. Goodman, P. S. Henry, and V. K. Prabhu, "Frequency-hopped multilevel FSK for mobile radio," Bell System Technical Journal, vol. 59, pp. 1257-1275, September 1980.

[7] L.-L. Yang and L. Hanzo, "Residue number system assisted fast frequency-hopped synchronous ultra-wideband spread-spectrum multiple-access: A design alternative to impulse radio," IEEE J. on Select. Areas in Commun., vol. 20, pp. 1652-1663, Dec. 2002.

[8] M. K. Simon, S. M. Hinedi, and W. C. Lindsey, Digital Communication Techniques - Signal Design and Detection. New Jersey: Prentice Hall 1995. 